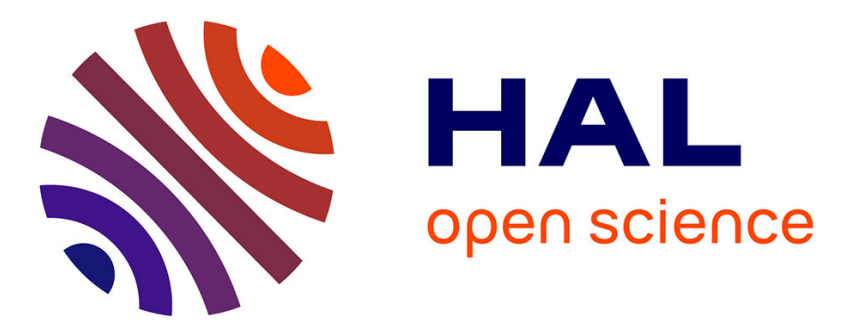

\title{
Towards context-aware assistive applications for aging in place via real-life-proof activity detection
}

\author{
Loïc Caroux, Charles Consel, Lucile Dupuy, Hélène Sauzéon
}

\section{To cite this version:}

Loïc Caroux, Charles Consel, Lucile Dupuy, Hélène Sauzéon. Towards context-aware assistive applications for aging in place via real-life-proof activity detection. JAISE - Journal of Ambient Intelligence and Smart Environments, 2018, 10 (6), pp.445 - 459. 10.3233/AIS-180505 . hal-01921226

\section{HAL Id: hal-01921226 \\ https://hal.inria.fr/hal-01921226}

Submitted on 14 Nov 2018

HAL is a multi-disciplinary open access archive for the deposit and dissemination of scientific research documents, whether they are published or not. The documents may come from teaching and research institutions in France or abroad, or from public or private research centers.
L'archive ouverte pluridisciplinaire $\mathbf{H A L}$, est destinée au dépôt et à la diffusion de documents scientifiques de niveau recherche, publiés ou non, émanant des établissements d'enseignement et de recherche français ou étrangers, des laboratoires publics ou privés. 


\title{
Towards context-aware assistive applications for aging in place via real-life-proof activity detection
}

\author{
Loïc Caroux ${ }^{\mathrm{a}, \mathrm{b}, *}$, Charles Consel ${ }^{\mathrm{b}, \mathrm{c}}$, Lucile Dupuy ${ }^{\mathrm{b}}$ and Hélène Sauzéon ${ }^{\mathrm{b}, \mathrm{d}}$ \\ ${ }^{a}$ CLLE laboratory, University of Toulouse Jean Jaurès \& CNRS, 5 Allées Antonio Machado, 31058 Toulouse, \\ France. \\ b Inria Bordeaux Sud-Ouest, 200 avenue de la vieille tour, 33045 Talence, France. \\ c Bordeaux INP, 1 avenue du Dr Albert Schweitzer, 33402 Talence, France. \\ ${ }^{\mathrm{d}}$ HACS laboratory, University of Bordeaux, 146 rue Léo Saignat, 33076 Bordeaux, France.
}

\begin{abstract}
Assisted living applications can support aging in place efficiently when their context-awareness is based on a reallife-proof approach to activity detection. Recently, Caroux et al. proposed a new approach to monitoring activities dedicated to older adults, named "activity verification". This approach uses a knowledge-driven framework that draws from the literature on older adults. The purpose of the present study is to address the limitations of this approach by scaling it up and by demonstrating that it is applicable to context-aware assistive applications for aging in place. First, an experimental study was conducted in which this approach was used to monitor a large range of daily activities, for a long period (8 weeks of experimentation) and involving several participants (7 participants). Second, this approach was used to validate two examples of context-aware assisted living applications, via simulation, based on real-life sensor log data. Results showed that the applicability of the "activity verification" approach scales up to a large range of daily activities by extending this approach (with accuracy values ranging between 0.82 and 1.00 depending on the activity of interest). Its inter-participant and intra-participant consistencies were demonstrated. Its limitations were addressed and the applicability to context-aware assistive applications for aging in place running on a dedicated platform was demonstrated.
\end{abstract}

Keywords: Assisted Living; Older adults; Activity Recognition; Routines; Activity Verification

\section{Introduction}

Independent living for older adults is a fundamental objective to promote aging in place, which has become a major societal challenge ${ }^{1}$. Indeed, people live longer in their home, even with numerous difficulties in their daily activities [1]. Pervasive computing support for assisted living, especially for older old adults, has become necessary since the global cost of care for these individuals, who can face cognitive impairment, has become more and more unsustainable [2,3]. In addition, there is increasing evidence that environmental support can be an effective approach to helping older adults perform their daily activities [4-6]. According to the environmental support framework [7], assistive technologies can be defined as all forms of technical support, that either provide an adaptation of the environment to make it more accommodating for persons with cognitive impairments or experiencing cognitive decline, or that equip persons with the means to compensate for their impairments. It has been argued that assistive technologies, especially technologies designed for cognitive impairment, have the potential to reduce disabilities of older adults [8].

The current challenge is to leverage advances in pervasive computing to design context-aware assistive

\footnotetext{
*Corresponding author. E-mail: loic.caroux@univ-tlse2.fr.

${ }^{1}$ In particular, several research projects that deal with ambient assistive living (AAL) are supported by the European Union. See the following website for more details and a comprehensive list of funded projects: http://www.aal-europe.eu
} 
applications that could improve the user's everyday functioning, as well as their caregiving network, towards improving well-being and prolonging aging in place. Monitoring Activities of Daily Living (ADLs) to ensure that they are performed is a decisive factor to achieve this objective. ADLs are abilities defining the functional status of an individual. This status can be assessed by professionals, such as occupational therapists, who can ensure that ADLs (e.g., self-care activities) are performed, in a suitable manner (e.g., time of the day, duration), and without deterioration over time. Results of this assessment contributes to determine what kinds and what levels of assistance are needed for an individual in their daily life to stay at their home or to be in a senior home.

Many approaches exist in the literature to monitor activities [9-11]. However, most of them aim to infer activities 1) without using prior knowledge about the user (e.g., [9]), 2) targeting heterogeneous age groups (e.g., [12]), 3) assuming multiple occupants in a home (e.g., [13]), and/or 4) deploying numerous sensors (e.g., [12]), including intrusive ones, such as cameras (e.g., [13]).

Recently, Caroux et al. proposed a new approach, namely the activity verification approach, to monitoring activities dedicated to older adults [14]. This approach uses a knowledge-driven framework that draws from the literature on older adults. It is centered on the user and the characteristics of their daily activities. This is particularly relevant for older adults because as they age, their daily activities are increasingly routinized to optimize their daily functioning [15]. Thus, these authors propose that users declare their daily routines, allowing a monitoring system to simply verify them, as opposed to inferring activities. A byproduct of their approach is that it requires a lightweight sensor infrastructure, comprising non-intrusive, low-cost, wireless devices. Verification is performed by applying a simple formula to sensor log data, for each activity of interest. The activity verification approach was validated with an experimental study, involving four participants, monitored during five days at their home. These preliminary results are very promising, while raising a number of research questions that need to be addressed to take this approach one step further.

First, is activity verification applicable to a range of ADLs, meaningful for determining the functional status of older adults? Indeed, this approach has only been applied to three ADLs (breakfast preparation, getting dressed and taking a shower). Second, does activity verification show intra-participant consistency over a long period of time? The existing results are only based on five days of sensor log data. Third, does activity verification show inter-participant consistency? Only four participants were included in their experimental study. Fourth, is activity verification as reliable as human observers to assess whether an activity is performed or not? Only one human observer judged whether the participants performed the activities of interest. Fifth, what are the potential applications of activity verification to support aging in place and how can their usefulness be assessed?

The contributions of this paper are as follows.

1. The activity verification approach is scaled up by a) designing formulas for new ADLs, namely getting up from/going to bed, lunch preparation and dinner preparation, and improving the formula for the getting dressed activity, b) monitoring activities for a long period to show intra-participant consistency of the approach (8 weeks of experimentation), c) involving several participants to demonstrate inter-participant consistency of the approach (7 participants), and d) guaranteeing the reliability of the approach by comparing results of the experiment and observations of three human judges.

2. Activity verification is put into practice by proposing context-aware applications that support aging in place. Two examples of applications are presented. The first one reminds the user of missed activities with respect to declared routines. The second one proposes to send a report assessing the activities of the user to a caregiver or a health professional. Both applications have been run on sensor log data to assess their resulting user interactions.

\section{Related work}

First the literature on aging and ADLs is reviewed. This review provides a fundamental understanding of the impact of aging on ADLs and suggests approaches to support them. Second, the research in monitoring activities is summarized along three dimensions: the settings, the types and number of sensors, and the accuracy of the monitoring. Lastly, the existing approaches of sensor-based activity recognition in the literature are introduced.

\subsection{Older adult daily activities}

Studying ADLs at home is particularly critical for the domain of aging in place $[16,17]$. The independent 
performance of ADLs is a necessary condition for autonomy and independence in everyday life [18]. A major consequence of cognitive decline in older adults is the progressive loss of the ability to perform ADLs. ADLs include Basic (BADLs) and Instrumental (IADLs) Activities of Daily Living [19]. BADLs refer to basic physical self-care tasks e.g., dressing, grooming, toileting, feeding), while IADLs are cognitivelycomplex, self-care tasks, such as meal preparation, medication and financial management.

In general, ADLs performed at home can be qualified according to several criteria: location in the home, time of the day, duration of the activity, actions required to perform the activity, sequence of these actions, and duration between these actions [20]. For older adults, these activities are well structured [21], involving sequential steps that tend to be "compiled" by older adults as a skill [22]. The accumulated reproduction of solutions, as well as aging related loss, tends to explain why older adults have a preference for routines. This phenomenon is called age-related routinization [15]; it precludes multiple activities to be conducted simultaneously.

Routinization of older adults can also be observed for sequences of activities within a day or across a week [23]. Thus, additional criteria for ADL performance of older adults can be used to qualify the interrelationship between activities: sequence order of the activities during a day and duration between the performances of these activities [20].

\subsection{Monitoring activities of older adults at home}

\subsubsection{Setting}

A lot of research has been addressing the monitoring of activities. However, most works have taken place in a non-real-life setting: a home dedicated to experimental studies $[13,24]$. In the context of older adults, an unfamiliar setting is contradictory to a reliable assessment of activities. Indeed, as demonstrated by various studies (e.g., [19]), as their cognitive resources decrease, older adults tend to optimize the remaining ones by increasingly organizing operations of their activities according to a strict routine. As a consequence, asking older adults to perform activities in an unfamiliar setting compromises their optimization strategies. The resulting assessment of their activity performance may be unrelated to their actual ability to live independently [19].

\subsubsection{Range of sensors}

To monitor older adults in their own home (i.e., a naturalistic setting), the range of sensors that can be utilized is limited. Sensors have to be non-intrusive and respect the privacy of users to be accepted and adopted [25]. For example, objects interfering with the person's life, such as RFID tags, should be avoided because they impose constraints on the user that can result in inaccurate measurements $[11,26]$. Similarly, wearable sensors or cameras are considered by most users as intrusive in their daily life [11,27], even if sensors embedded in more common devices, such as smartphones, could be considered as more acceptable [28]. In contrast, simple sensors, such as motion-based sensors, are very reliable to measure activities in the home environment [13] and are better accepted by users [14].

Another issue is the number of sensors that can be installed in the older adults' home. Some approaches are data-driven relying on extensive measurements, based on numerous sensors, populating the entire home [29]. The ubiquitous nature of sensors, intrinsic to these approaches, represents a major obstacle to their acceptance by older adults, creating a feeling of stigmatization and invasion of privacy [30].

Obviously, limiting the number of sensors and forbidding cameras give a monitoring approach that is not as accurate as a human observer. However, in practice, this approach produces meaningful and actionable information.

\subsubsection{Accuracy of activity monitoring}

Researchers have proposed various granularity at which activities can be monitored. For example, Mihailidis et al. examine the various steps of handwashing [31]. Not surprisingly, the finer the granularity gets, the more complex the monitoring process becomes because of the amount and level of data to gather and analyze. This situation contrasts with home-based activity monitoring of older adults, where studies show that the granularity of the monitoring does not need to be fine-grained. More specifically, cognitive decline first impact the IADLs because they require high-level cognitive functions to initiate, plan and execute a task [19], compared to BADLs. Also, IADLs inherently involve numerous interactions with the environment to perform the sub-tasks of a given task (e.g., breakfast involves preparing coffee by opening specific doors of kitchen cabinets to get the coffee, a filter and a cup, and turning on the coffee 
maker) [32]. This situation allows tracking the execution of sub-tasks via interactions with sensor-equipped components of the environment.

\subsubsection{Knowledge-driven and data-driven activity monitoring}

Sensor-based activity recognition for assisted living is a recent but fairly studied topic in the literature [33]. There exist several approaches that can be categorized within data-driven or knowledge-driven approaches [34].

Data-driven approaches use statistical and probabilistic methods to learn activity models from datasets (e.g., machine-learning method). Data-driven approaches have many advantages. In particular, they allow the modeling of uncertainty and temporal parameters [35]. However, they may require that datasets come from large sets of sensors. The main consequence is that evaluating data-driven approaches beyond a fully controlled environment, such as a lab setting, may be complex. This may explain why there is no study yet in the literature, evaluating these approaches in a real-life environment, such as a home with a typical occupant, if not one with an older adult. This lack of real-life studies is illustrated by Dawadi et al. [36,37] that report on the use of machine learning to monitor the wellbeing of individuals, but restrict their study to a lab setting.

Knowledge-driven approaches use existing domain knowledge and heuristics to create activity models. In practice, these approaches appear to be well suited to monitor activities of older adults. They use domain knowledge, i.e. they are centered on the user and the characteristics of their activities [34]. One advantage is that they do not require a large set of sensors.

Recently, Caroux et al. proposed an approach to verifying activities that is driven by knowledge on older adults [14]. This approach, named activity verification, is detailed in the next section.

Note that a detailed literature review of data-driven and knowledge-driven approaches is given by Rafferty et al. [34].

\section{Real-life-proof activity verification}

This section introduces the activity verification approach and presents how it can be scaled up and validated to be usable for context-aware assistive applications. Scaling up mainly implies to widen the range of target activities. Validating involves assessing the robustness and reliability of the approach with a greater number of participants (inter-participant consistency) and for a longer period of experimentation (intra-participant consistency) than was done previously.

\subsection{Introducing activity verification methodology}

This section presents the activity verification methodology [14]. For the remainder of the paper, this approach and methodology are referred as activity verification.

\subsubsection{Preliminaries}

The activity verification methodology is dedicated to monitoring a single occupant in their home. Measurements of user interactions with the environment mainly rely on three types of sensors: motion sensors (to detect movement in a room), contact sensors (to detect the opening of doors, drawers and cabinets) and smart switches (to detect whether an appliance is in use). The activity verification methodology relies on a definition of activity that draws from the literature on aging:

An activity 1) is situated in a room, 2) entails routinized interactions of the older adult with the physical environment, and 3) is performed one at a time.

\subsubsection{Methodology}

We are now ready to present the main steps of the activity verification methodology, as they were followed for the experimental study described later.

Routine declarations. An experimenter, trained in ergonomics, visits each participant at their home and asks them to sketch each activity of interest. The outcome is a list of markers, characterizing an activity (e.g., breakfast involves using a given appliance, opening a drawer, etc.), as well as a schedule of every day's activities of interest (e.g., breakfast takes place between 7:00AM and 9:00AM). Markers should be robust (high degree of certainty) and unique in a given context (e.g., coffee is only made once in the morning)

Sensor placement. Markers are then examined to determine whether they match a type of sensor and, if so, where sensors should be placed. As such, determining markers and selecting sensors are intimately dependent phases.

Routine formulas. A formula models whether a given activity is performed. It is defined with respect to the declared routine and underlying sensorequipped markers. A formula is thus knowledge driven in that it relies on the fundamental attributes of 
an activity, mentioned in the above definition. A formula verifies an activity by producing a score, whose value ranges between 0 and 1 . The value 0 means that the activity has not been performed, according to the user's routine. The value 1 indicates that the sensed measures strictly match the user's routine.

To sum up, formulas model activities of interest. They are applied to the sensor log data that record specific environment interactions. In doing so, formulas automatically verify whether some interactions have occurred according to the declared routines.

\subsubsection{Limitations}

We identified four main limitations in the preliminary validation of the activity verification approach [14]. First, there were too few activities that were considered: the approach was only applied to three ADLs (breakfast preparation, getting dressed and taking a shower). Second, the intra-participant consistency of the approach was not tested over a long period of time: results were based on five days of sensor log data. Third, the inter-participant consistency was not properly tested: only four participants were included. Fourth, the reliability of the approach was only partially tested: a unique human observer judged whether the participants performed the activities of interest.

\subsection{Scaling up activity verification}

In this section, we scale up the activity verification approach. First, the range of target activities is widened. To do so, the notion of activity is extended, improving the modeling of BADLs. This extended version of activity is then illustrated by concrete examples. Finally, new routine formulas are introduced and all these ingredients are put together.

\subsubsection{Widening the range of activities}

The present study covers the most critical daily activities for older adults. Three BADLs are studied in the present study: getting up from/going to bed, getting dressed and taking a shower. One IADL is also targeted: meal preparation, which is performed three times a day (breakfast, lunch, and dinner). The main reasons to choose these activities are as follows. First, they are among the activities that are sensitive to agerelated functional decline [19], as well as routinization [38]. As a result, there is a rich collection of articles reporting on the monitoring of these activities (e.g., $[10,26])$. Second, they allow exercising many dimensions of the present approach, illustrating different sensing functionalities, locations, and activity requirements.

\subsubsection{Extending the notion of activity}

According to the activity verification methodology, an activity is examined individually. However, some activities may not involve interactions with the environment that are unique. This situation is illustrated in the preliminary validation of the approach with the activity of getting dressed: it relies on a single marker, typically the door of a wardrobe. The problem is that this marker is not unique because the door can potentially be open many times a day.

To strengthen the notion of activity, it is extended with an additional property introduced by Chen et al. [20]: the interrelationship between activities. Specifically, determining whether an activity has been performed depends on whether it is preceded or followed by another activity. Furthermore, the time between the occurrences of the activities of interest also contributes to characterizing a given activity.

Following the activity verification methodology, this routinized sequence is declared by the older adult. For example, Ms. Dupont, a fictional participant, describes her daily routines to the experimenter as follows: after getting up from bed, she usually goes to her kitchen to prepare and take her breakfast, then she takes a shower, and finally gets dressed. Later in the day, she takes her lunch and her dinner, and finally she goes to her bathroom for a short moment before going to bed. She also describes that all of these activities are made at regular times during the day. Finally, she mentions that she only takes a shower once every two days, considering her physical condition.

Note that a range of activities can be verified without relying on the occurrence of other activities. However, as we widen the scope of activity verification, some activities require more contextual information.

\subsubsection{Examples of extended activities}

The extended notion of activity can be illustrated with concrete examples that require the interrelationship property. First, consider the activity of getting dressed. Its verification can consist of ensuring that the wardrobe door opening detection is preceded or followed by the detection of an activity in the bathroom, depending on the person's routine. Similarly, the activities of getting up from/going to bed could not be verified with a single marker (e.g., a presence in the bedroom in the morning or in the evening). The activity of getting up from bed needs to be followed by an 
activity in another room (e.g., after she wakes up, Ms. Dupont goes to the kitchen to make a coffee). Going to bed can be verified by a preceding activity in another room, such as the bathroom.

\subsubsection{New routine formulas}

We now introduce new routine formulas, demonstrating that activity verification scales up. Note that one dimension of the scalability of the present methodology is demonstrated by the fact that the activities of interest are uniquely captured by the proposed formulas, which in effect abstract over inter-individual variations in performing routines. Only markers of interest have to be customized with respect to the older adults' routines.

\section{Meal preparation}

First, the breakfast preparation initial formula is generalized to make it applicable to lunch and dinner. This generic meal preparation (MP) formula is defined in Eq. (1).

$$
S c^{M P}=T^{M P} \times \frac{M^{M P} \times 4+\frac{s_{1}^{M P}+\cdots+S_{n}^{M P}}{n}}{5}
$$

Where $S c^{M P}$ is the score for the activity of meal preparation. This formula reflects the constraint that meal preparation occurs at a specific time of the day $T^{M P}$ (e.g., between 6:00AM and 11:00AM for breakfast), which takes value 1 , if it is within the expected time frame of the day, and value 0 , otherwise. Furthermore, the formula accounts for the fact that meal preparation often includes a major marker, corresponding to the use of a specific appliance or cabinet $M^{M P}$. To translate the importance of this marker, its value is weighted with a factor of 4 . The weight factor of 4 was calculated a posteriori in order to ensure the predominance of the major marker in the final value. It is summed with the rest of the markers of the meal preparation activity, which are averaged $S_{i}^{M P}$. The result of this sum is then divided by 5 to obtain a final value between 0 and 1 . As can be noted, duration is ignored in the formula because this activity consists of a few markers that are to be validated over the time of the preparation.

This generic meal preparation formula can then be instantiated for specific meals. For example, instantiating it for breakfast may involve giving a time frame as mentioned above, in addition to defining the coffee maker as a major marker and doors of the kitchen cabinets as other markers, containing mugs and coffee. For lunch and dinner, the generic meal preparation formula can be instantiated with respect to a time frame and a major marker defined as the drawer containing the silverware.

\section{Taking a shower}

As explained previously, BADLs involve fewer actions that can be sensed than IADLs. However, the duration of a particular event, or a specific sequence of events can be exploited. The formula of the activity of taking a shower (TS) is defined in Eq. (2).

$$
S c^{T S}=T^{T S} \times D^{T S}
$$

The time of the day is again relevant in this formula. This activity requires to be performed over a minimal period of time. As a consequence, $D^{T S}$ takes value 1 , if this minimal duration is reached, and value 0 otherwise. This activity relies on a unique marker that corresponds to a motion detector, placed over the shower.

\section{Getting dressed}

Regarding the activity of getting dressed (GD), as mentioned previously, the original formula is extended: it involved a unique marker (e.g., wardrobe door); it now relies on an interrelationship with another activity. The new formula is defined in Eq. (3).

$$
S c^{G D}=T^{G D} \times M_{1}^{G D} \times M_{2}^{G D}
$$

Where $M_{1}^{G D}$ is the major marker of this activity (e.g. wardrobe door opening), which has value 1 if the sensed interaction occurred, and value 0 otherwise. Furthermore, $M_{2}^{G D}$ is the associated marker, such as an activity in bathroom, preceding or following the major marker by at most 5 minutes. This associated marker takes value 1, if the event occurs and matches the constraints on sequencing and timing; it takes value 0 otherwise.

\section{Getting up from / going to bed}

The formulas for the activities of getting up from/going to bed require an associated marker, such as the one for the activity of getting dressed. Specifically, the formula for the activity of getting up (GU) is defined in Eq. (4).

$$
S c^{G U}=T^{G U} \times M_{1}^{G U} \times M_{2}^{G U}
$$

Where $M_{1}^{G U}$ is the major marker of this activity (activity in the bedroom), and $M_{2}^{G U}$ is the associated marker (e.g., presence in kitchen in the following 10 minutes).

The formula for the activity of going to bed (GB) is defined in Eq. (5).

$$
S C^{G B}=T^{G B} \times M_{1}^{G B} \times M_{2}^{G B}
$$


Where $M_{1}^{G B}$ is the major marker of this activity (presence in the bedroom), and $M_{2}^{G B}$ is the associated marker (e.g., presence in bathroom in the preceding 10 minutes).

The present approach focuses on specific activities of daily living that are well-known to determine the status of an older adult, as opposed to attempting to model as many activities as possible. The latter objective would require an arbitrary number of sensors, measuring an open-ended set of environment interactions. Additionally, detecting arbitrary activities would likely require a richer framework to define formulas than the one described in this paper, including a range of tools to author formulas. In contrast, the proposed approach focuses on providing a methodology to capture inter-individual variability via routine formulas.

\subsection{Experimental validation}

An experimental study was designed to validate the scaled-up, activity verification methodology. The goal of the experiment is to assess the robustness and reliability of this methodology by verifying a larger range of activities, involving a greater number of participants, for a longer period of experimentation.

\subsubsection{Participants}

To test the present research assumptions, it is critical to include community-dwelling, very old adults. To do so, the study was done in collaboration with a public home care service for older adults, which provided access to the medical file of their beneficiaries. As described in Table 1, 7 participants, aged 82.0 on average ( $\mathrm{SD}=5.80$ ) were recruited according to specific exclusion criteria: dependency syndrome, neurological or musculoskeletal disease or systemic disorders. The main inclusion criterion was cognitive integrity with an MMSE score greater than 24 [39]. According to the Helsinki declaration (WMA, 2008), approval was sought and obtained from the ethics committee of the University of Bordeaux. As well, a request for the study was sent to the CNIL (the "French Commission on Informatics and Human Rights") regarding the privacy of the logged data; this request got approved. All participants provided a written consent form prior to their participation.

Their functional status for some activities of daily living was assessed. First, their performance in IADLs was evaluated, using the time-based IADL assessment test [40]. Specifically, this test consists of asking a par-
Table 1

Participant profiles

\begin{tabular}{ll}
\hline Participant profile & Mean (SD) \\
\hline Age & $82.0(5.80)$ \\
Gender & 6 women and 1 man \\
Education years & $9.71(1.60)$ \\
Family status & all widowed \\
MMSE [0 - 30] & $27.14(0.90)$ \\
Time-based IADL [5 - 15] & $5.29(0.76)$ \\
Self-reported IADL [9-45] & $12.14(4.38)$ \\
Routinization [0 - 40] & $15.86(5.55)$ \\
\hline Note. SD=Standard Deviation. Interval notations are used \\
\multicolumn{2}{r}{ for score ranges. }
\end{tabular}

ticipant to perform an activity with a time limit. If the activity is achieved without error and without exceeding the time limit, a score of 1 is given. A score of 3 means that the participant has major difficulties to perform the activity. The participants were tested on five different activities; this gave scores ranging from 5 (ideal performance) to 15 (major difficulties). They were also asked to self-assess their functional status, using the 9-item IADL scale [41]. For each item, the participant assessed their performance: 1 denotes no difficulties and 5 denotes major difficulties. Finally, the degree of routinization of the participants was evaluated using the routinization scale defined by Bouisson [23]. Based on the results of these tests (see Table 1), the participants performed their ADLs well and had a high level of autonomy, although they perceived themselves as experiencing difficulties. The participants showed a variety of routinization degrees, which plays a key role to assess whether the activity verification approach covers a wide spectrum of behaviors.

\subsubsection{Data collection}

Logs of the sensors placed in the participants' homes were collected during 8 weeks (i.e., 56 days). The same set of sensors was placed in the home of all the participants. However, they were not necessarily used the same way to monitor the activities of interest. For example, participants may or may not take milk from the fridge to make breakfast; they may use a coffee maker or a microwave to prepare a hot drink.

Sensor logs consist of the sensor identifier, its status, and a timestamp. The sensor identifier corresponds to a sensor type (motion detector, contact sensor and smart switch) and its location. We selected the logs pertaining to the sensors, located in the rooms, corresponding to the activities of interest (namely, kitchen, bedroom, and bathroom). 


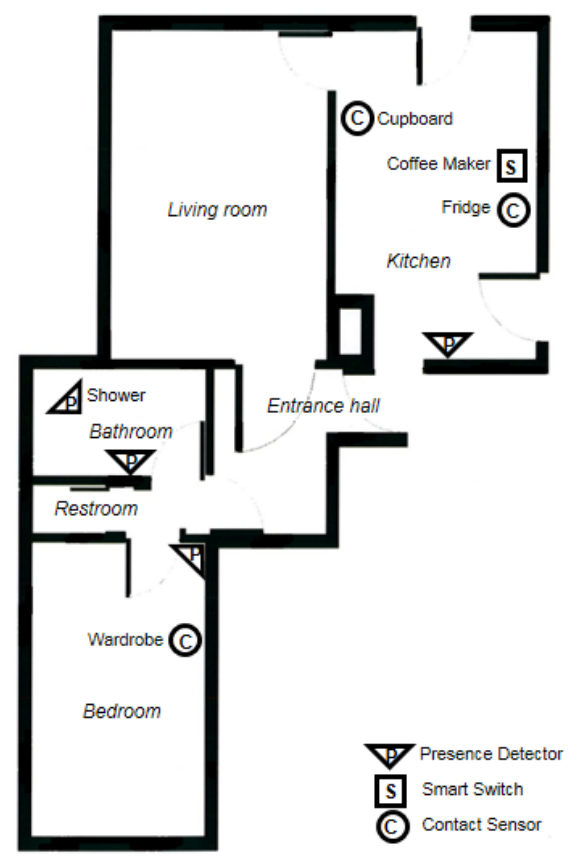

Fig. 1. Example of an apartment layout with sensors

Figure 1 shows an example of an apartment layout, populated with sensors corresponding to the activities of interest and related rooms. In this example, sensors in the kitchen are placed in the following way. A smart switch detects whether the coffee maker is used. Two contact sensors detect whether the cupboard or the fridge are open. Note that the same sensors may be placed differently in other participants' home. For example, the smart switch can be used to detect the usage of the microwave; the contact sensors can be used to detect the opening of the cutlery drawer or the fridge. Again, the sensor placement is strictly dependent on the activity routines of the user. Also, note that the sensors are placed in such a way as to ensure that only a given behavior is detected. For example, the motion detector placed in the shower is directed so as to detect the presence of the user only if they are actually inside the shower cubicle. At the beginning of the data collection, the sensor installer checks whether the motion detector of the shower exhibits the expected behavior.

The present experiment was designed to be as inexpensive as possible, in terms of cost of material, cost of sensor installation and cost of monitoring of data collection. The sensors we used are widely available, easy to configure, and low cost. The experimenter could easily install the sensors by themselves in the house of the participants. The sensors were connected wirelessly to a gateway, which communicates with our server via the Internet. Sensors’ data were collected and stored automatically and continuously. The proper functioning of the sensors and data collection could be remotely monitored by the experimenter at any time.

\subsubsection{Data analysis}

\section{Routine formulas application}

The same set of routine formulas, defined in the previous section, was applied to sensor log data recorded during 56 days, across the 7 participants. Although the formulas were the same for all participants, the markers were customized with respect to each participant's routines. For example, breakfast preparation involves a smart switch associated with the coffee maker for a given participant. While, the major marker is a contact sensor placed on the door to a specific kitchen cabinet, for another participant.

For each participant and for each day, applying the routine formulas determines whether each activity of interest is detected, i.e., whether the sensed measures match the participant's routines. Figure 2 displays an example of activity verification results for one of the participants over the 56 days of the experiment. Each dot displayed in the graph means that a given activity was detected on the corresponding day and time of the day. In this figure, all the activities took place between 5:00AM and 2:00AM. Each day, activities are generally performed in the same order. Getting up from bed, breakfast preparation, taking a shower and then getting dressed are performed in the morning. Lunch preparation is performed at midday, and dinner preparation early in the evening. Finally, the participant goes to bed in the evening or late at night. Note that the results displayed in Figure 2 are obtained automatically by executing the routine formulas, without any intervention from the human observer.

Occasional losses of data were observed during the experiment due to different kinds of technical problems (Internet outage, sensor failure, etc.). Consequently, some fragments of logs were missing and could not be used to verify whether some activities were performed. To facilitate data analysis, the detection of an activity was extrapolated wherever a data loss occurred in the relevant period of the day. The time of the day of this extrapolated detection of activity was calculated by averaging the times when the same activity was performed in the previous and the following days. For example, during the experiment in Ms. Dupont's home, logs were missing on day 45, between 5:00PM to 10:00PM, due to an Internet outage. Ms. Dupont declared that she prepares her dinner be- 


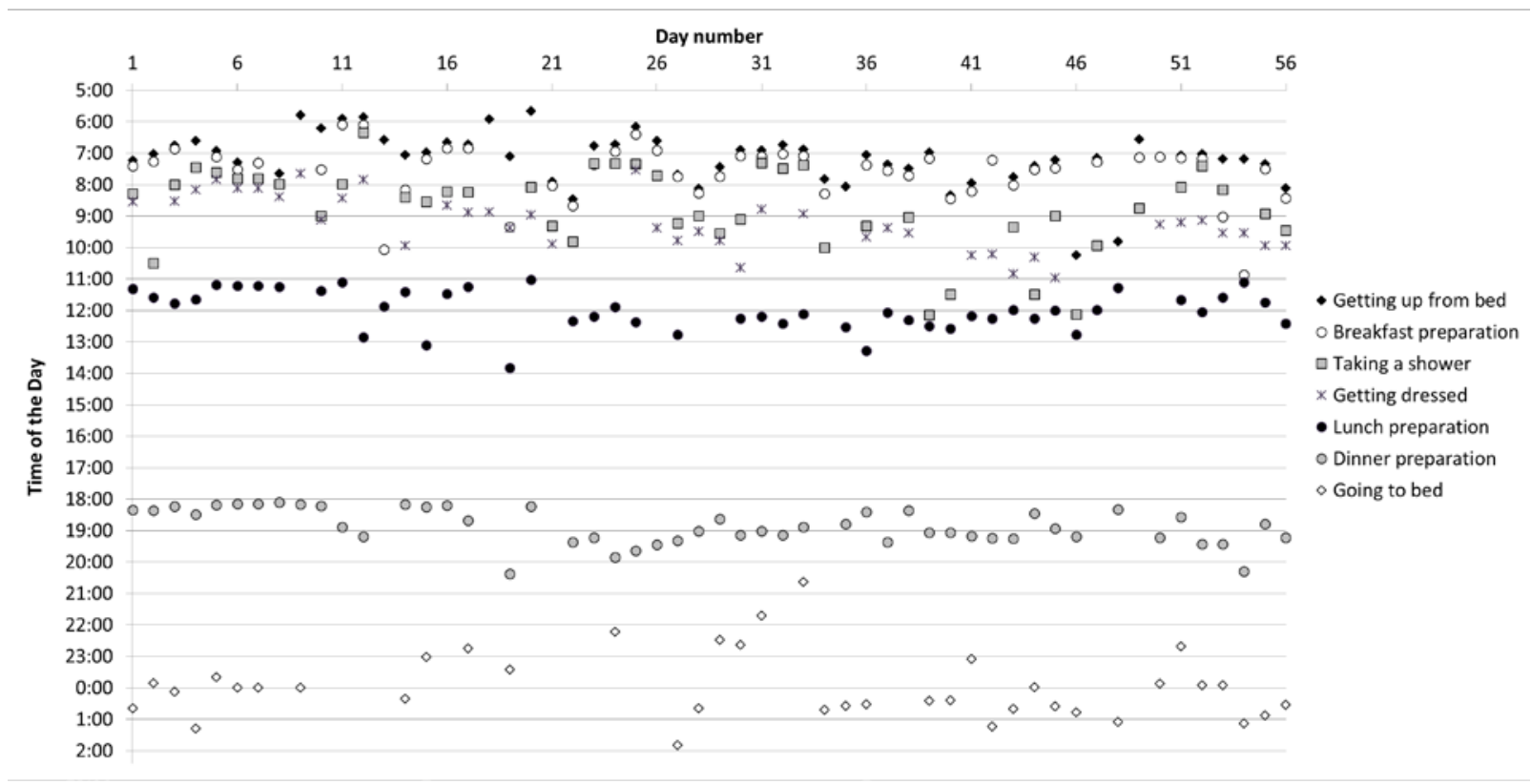

Fig. 2. Activities of a participant over a period of 8 weeks.

tween 5:00PM to 10:00PM, due to an Internet outage. Ms. Dupont declared that she prepares her dinner between 6:00PM and 8:00PM, and in fact, this activity occurred at $6: 20 \mathrm{PM}$ on day 44 , and at $6: 40 \mathrm{PM}$ on day 46. As a result, it was extrapolated that Ms. Dupont performed the activity of dinner preparation on day 45 at 6:30PM. For each participant, 24 detections of activity performance were extrapolated on average (SD $=10$, Min = 9, $\operatorname{Max}=35$ ). This situation corresponds on average to $6.3 \%$ of the total number of detections of activity performance $(\mathrm{SD}=2.6$, $\mathrm{Min}=2.3 \%$, $\mathrm{Max}$ $=8.9 \%$ ).

Sensitivity indices

The results of applying the routine formulas are now analyzed on the log data of the 7 participants. The accuracy of the formulas is tested with the calculation of two specific indices: the sensitivity and the response bias indices, respectively $A$ ' and $B{ }_{D}$ for nonparametric data [42].

Sensitivity indices are used in Signal Detection Theory to measure performance in Yes/No tasks (see [43]). To do so, participants of such tasks discriminate signals (stimulus is present) and noises (stimulus is absent). In the presence of a stimulus, Yes responses are correct and termed hits. In the absence of a stimulus, Yes responses are incorrect and termed false alarms. Then, rates of hit and false alarms are used to calculate the indices. $A$ ' measures the ability of the participant to correctly discriminate the presence or the absence of a stimulus. This index is contained between 0 (extremely low sensitivity) and 1 (extremely high sensitivity). $B{ }_{D}$ measures the general tendency of the participant to respond Yes or No. $B{ }_{D}$ is contained between -1 (tendency to respond Yes and produce false alarms) and 1 (tendency to respond No and miss stimuli).

In the present experiment, the formulas take the role usually played by human participants in Yes/No tasks. Twenty-one sets of sensor logs were randomly selected from the data collected at participants' home (three per participant); they covered an entire day. The present version of the Yes/No task was conducted as follows. First, three naive human observers were recruited, to judge whether the participants performed the seven activities of interest. To do so, the human observers were given information about the sets of sensors deployed in the participants' houses and the participants' declarations of daily routines. Then, scores of activities were computed using the formulas. The sensitivity indices $A$ ' and $B{ }^{\prime}{ }_{D}$ were calculated three times, from the results of each of the three human observers. In doing so, interjudge reliability was achieved. Table 2 displays the three different sets of values $A$ ' and $B{ }^{\prime}{ }_{D}$, obtained for each activity of interest. In addition, the agreement between the three human observers was reported using Fleiss' Kappa interrate reliability statistic for each activity of interest. 
Table 2

Sensitivity indices values

\begin{tabular}{lcccccccc}
\hline Index & \multicolumn{3}{c}{$A^{\prime}$} & \multicolumn{3}{c}{$B^{\prime \prime}{ }_{D}$} & Fleiss' Kappa \\
\hline Human observer & H1 & H2 & H3 & mean & H1 & H2 & H3 & \\
\hline Getting up from bed & 1.00 & 1.00 & 1.00 & 1.00 & 0.00 & 0.00 & 0.00 & 1.00 \\
Getting dressed & 1.00 & 0.85 & 0.84 & 0.90 & 0.00 & -0.89 & -0.87 & 0.42 \\
Taking a shower & 0.84 & 0.91 & 0.88 & 0.88 & 0.23 & 0.91 & 0.18 & 0.61 \\
Breakfast preparation & 0.99 & 0.79 & 0.79 & 0.86 & 1.00 & -0.93 & -0.93 & 0.41 \\
Lunch preparation & 0.87 & 0.83 & 0.81 & 0.84 & -0.95 & -0.94 & -0.93 & 0.52 \\
Dinner preparation & 1.00 & 0.83 & 0.83 & 0.89 & 0.00 & -0.95 & -0.95 & 0.68 \\
Going to bed & 0.96 & 0.50 & 1.00 & 0.82 & -0.31 & 0.00 & 0.00 & 0.20 \\
\hline
\end{tabular}

Four types of results were observed. First, regarding the activity of getting up from bed, all the scores produced by the routine formula perfectly match the judgments of the three observers, i.e., $A^{\prime}=1.00$ (mean $A^{\prime}$ $=1.00$ ). The formula can be considered as extremely sensitive.

Second, most of the scores produced by the formulas for getting dressed (mean $A^{\prime}=0.90$ ), breakfast preparation (mean $A^{\prime}=0.86$ ), lunch preparation (mean $A^{\prime}=0.84$ ) and dinner preparation (mean $A^{\prime}=0.89$ ) match the judgments of the three observers. The formulas can be considered as highly sensitive. The response bias indices indicated that these formulas were liberal (i.e., the formulas have a tendency to respond Yes). This situation means that these formulas may seldomly produce false alarms, i.e., detect activities that were not actually performed.

Third, results for taking a shower showed that most of the scores of the formula match the judgments of the three observers (mean $A^{\prime}=0.88$ ). The formula can also be considered as highly sensitive. The response bias indices indicate that this formula is slightly conservative (i.e., the formula has a tendency to respond No). This situation means that this formula may seldomly miss stimuli.

All three types of results presented so far are quite consistent in the sensitivity indices across the three human observers' judgments. The $A$ ' index of accuracy was quite similar for each activity, with a mean $A$ ' that is equal to or tends towards 1.00 , and the $B{ }_{D}$ index showed a bias in the same direction. The Kappa values for these activities range from 0.41 and 1.00 , which means that the agreement between the observers is from "moderate" to "almost perfect".

The fourth type of result did not follow this consistency: the activity of going to bed showed different indices of accuracy as judged by the three human observers, even if the mean $A^{\prime}$ is high (mean $A^{\prime}=0.82$ ). Two of them (H1 and H3) delivered judgments that match all or most of the scores produced by the corresponding formula. However, the accuracy was weaker for the other observer (H2). Depending on the judgment of the human observer used, the formula can be considered as highly or moderately sensitive. There is more consistency regarding the index of bias. The index indicates that the formula has no bias, or may be slightly liberal, as compared to the judgments of the three human observers. The Kappa value for this activity (0.20) indicates a slight agreement between the observers.

In summary, the present experimental study has validated the scaled-up, activity verification methodology. The new routine formulas, as well the original ones, are accurate in that they have almost always detected when an activity of interest was present in a given log data, as compared to the judgments of the three naive observers. This has been observed for a large range of activities, with more participants, and a longer period of data collection; results of routine formulas have been compared with a greater number of human observers to achieve interjudge reliability.

\section{Context-aware assisted living applications}

In this section, two examples of dedicated assistive application are presented. They are based on activity verification that would allow coordinating caregiving tasks and regulating the caregiver's load. This approach promotes a behavioral intervention, rather than a pharmacological intervention, towards improving well-being and prolonging aging in place. Two examples of applications are presented in this section: the first application reminds the user about missed activities; the second application reports an assessment of the user's activities to a caregiver. Both applications were simulated on sensor log data, collected during the experimental study, to assess their practical nature. 


\subsection{Notifications for self-regulation}

The well-being of older adults, especially with cognitive impairment, relies on the fact that daily activities can be accomplished independently [44]. To support this aspect, some applications could help the users to conform to their daily routines via activity monitoring. When an activity is not detected within a given period of time in the day, a message could be displayed (e.g., on a tablet or on TV) to remind the user about an activity. In a user-centered design framework, this kind of assistive application should be designed according to the user needs $[45,46]$. For example, Ms. Dupont usually prepares her dinner between 6:30PM and 8:00PM. One day, she does not prepare her dinner because she has a cold and has lost her appetite. At the end of this period of time (i.e., after 8:00PM), a notification reminds her to prepare dinner.

The results of activity verification presented earlier were used to simulate the number of notifications that would be issued to the participants of the present study. We calculated the number of notifications that each of the 7 participants would have received on average each week and for each activity of interest.

Table 3 displays the results of the simulation for each participant. The number of activities included in the calculation of the score was personalized according to the participants' actual routines.

Results have shown that notifications for meal preparations and for getting up activities are marginal. That means that these activities are almost always detected. In contrast, notifications for getting dressed, taking a shower, and going to bed (for two participants) occurred more often. That means that these activities are detected less frequently. This situation suggests several explanations. Regarding the activity of taking a shower, the routine declared by the participants was not as accurate as information given for other activities. This is probably due to the fact that participants declared a routine that is socially acceptable (e.g., everyday or every two days) rather than the actual one. This situation shows a limitation of a knowledgedriven approach based on user-supplied routine declarations.

Regarding the activity of getting dressed, the weak detection of this activity could be due to the location of the contact sensor in the bedroom. Specifically, when the sensor is placed directly on the drawer containing underwear, the activity is detected accurately. In particular, the mean number of simulated notifications for getting dressed of Participant P1 is the weak-
Table 3

Mean number of simulated notifications per week per participant

\begin{tabular}{|c|c|c|c|c|c|c|c|}
\hline Participant & $\mathrm{P} 1$ & $\mathrm{P} 2$ & P3 & $\mathrm{P} 4$ & P5 & P6 & P7 \\
\hline $\begin{array}{l}\text { Getting up } \\
\text { from bed }\end{array}$ & 0.0 & 0.1 & 0.1 & 0.3 & 0.3 & 0.1 & 0.1 \\
\hline $\begin{array}{l}\text { Getting } \\
\text { dressed }\end{array}$ & 1.9 & 5.4 & 5.3 & 2.1 & 5.0 & 3.4 & 2.1 \\
\hline $\begin{array}{l}\text { Taking a } \\
\text { shower }\end{array}$ & 1.4 & 2.3 & 2.3 & 4.3 & 6.1 & $\mathrm{n} / \mathrm{a}$ & $\mathrm{n} / \mathrm{a}$ \\
\hline $\begin{array}{l}\text { Breakfast } \\
\text { preparation }\end{array}$ & 1.0 & 0.6 & 0.3 & 0.4 & 0.1 & 0.6 & 0.1 \\
\hline $\begin{array}{l}\text { Lunch } \\
\text { preparation }\end{array}$ & 1.1 & 0.4 & 0.5 & 0.1 & 0.5 & 0.6 & 0.4 \\
\hline $\begin{array}{l}\text { Dinner } \\
\text { preparation }\end{array}$ & 0.8 & 0.3 & 0.1 & 1.1 & 0.3 & 0.4 & 0.5 \\
\hline $\begin{array}{l}\text { Going } \\
\text { to bed }\end{array}$ & 2.3 & 6.1 & 0.4 & 0.8 & 0.5 & 0.1 & 0.1 \\
\hline Total & 8.4 & 15.1 & 8.9 & 9.0 & 12.8 & 5.3 & 3.4 \\
\hline
\end{tabular}

Note. The activity of taking a shower was not monitored for participants P6 and P7.

est one because the sensor could not be placed on a drawer containing underwear exclusively.

Regarding the activity of going to bed, the associated marker of this activity (e.g., presence in the bathroom in the preceding 10 minutes) was systematically detected for all but two participants (P1 and P2). This suggests that another kind of marker should be investigated. For example, no light in the bedroom combined with no detection of presence in the other rooms could provide accurate results.

In any case, regardless the activity of interest, it is important to note that an absence of detection can be due to the fact that the person may be away from home. Also, the person may voluntarily not perform the activity. In fact, these situations show the limitation of any system of activity monitoring: Where does such a system stop supporting the user and starts compromising their self-determination?

Evidently, this kind of application must be personalized with respect to the needs and expectations of the monitored person to improve its efficacy. For example, the older adult may choose to be monitored and to receive notifications only for a few activities of interest. Furthermore, future works need to assess the acceptability of such notifications. Several research issues have been raised from the results of the simulation. How many notifications per day or per week are acceptable by an older adult? Does a notification during the evening or even at night (regarding the activity of going to bed) is acceptable? 


\subsection{Status reports to the caregiver}

The actions of the caregiving environment are often limited or impeded by the lack of a proxy at the older adult's home. Such a proxy is needed for a number of actions, including coordinating the planning of care services, gathering activity information of the older adult, reminding activities and appointments, monitoring potentially unsafe activities and situations. Assistive technologies have been proposed as an efficient means for reducing interpersonal tensions between caregivers and care-receivers [47], in addition to increasing independent activity, self-confidence, and the quality of care [48].

An application could send a report of daily and/or weekly routines of the older adult to the caregiver. The report could determine whether each activity of interest was performed during the given period of time (e.g., [49]). Of course, such an application should be designed according to the needs and expectations of the older adult and the caregiver, especially in terms of preferences, privacy, autonomy, and social participation [50].

Our experimental results on activity verification were used to simulate daily status reports to the caregiver. For each of the 7 participants of the study, 56 status reports were simulated. In this simulation, the status report was based on the number of activities that were correctly detected during the day. A global score was computed according to this number. An "A score" means that all activities of the person were detected. A " $B$ score" means that one or two activities were not detected. Finally a " $C$ score" means that three activities or more were not detected. Note that in the simulation the status report does not give any information, other than whether activities were detected. For example, Ms. Dupont needs (or wants) to be monitored for each activity but taking a shower. If all activities of interest are detected, the caregiver receives a status report with an $A$ score. If the dinner preparation is missed, the status report indicates a $B$ score. When no meal preparation is detected, the status report indicates a $C$ score.

Table 4 shows the results of the simulation for the 7 participants of the study. The number of activities included in the calculation of the score was personalized according to the participants' actual routines.

Obviously, results of status reports were in line with the results of notification. Some participants got status reports with lower scores when one or two of their activities were not accurately detected. In practice, the
Table 4

Number of simulated status reports by level of routine detection per participant

\begin{tabular}{lccccccc}
\hline Participant & P1 & P2 & P3 & P4 & P5 & P6 & P7 \\
\hline A score & 20 & 3 & 10 & 13 & 2 & 24 & 35 \\
B score & 27 & 35 & 43 & 38 & 48 & 32 & 21 \\
C score & 9 & 18 & 3 & 5 & 6 & 0 & 0
\end{tabular}

Note. The activity of taking a shower was not monitored for participants P6 and P7.

status report should be designed by taking into account the activities that are inaccurately detected for a given participant: they should be excluded from the score calculation. In the present simulation, one can imagine that if these kinds of activities were excluded (e.g., getting dressed, going to bed, etc.), the scores would be more reliable (e.g., $A$ instead of $B$ ).

The proposed application should be personalized with respect to the needs and expectations of the monitored user and the caregiver. This kind of application can be designed to send information to the family and/or professional caregiver, or even to the older adult. Although the data should be rendered depending on the knowledge that the receiver has about the older adult, the purpose may be the same: determining whether the older adult needs (more) assistance in their daily life. In such a case, one kind of assistance would be to notify the older adult whenever there is a deviation in their daily routine, as discussed earlier.

\section{Conclusions and future works}

In this paper, the reliability of the activity verification approach, proposed by Caroux et al. [14], has been improved. In particular, the activity verification approach was scaled up by designing formulas for new ADLs. Activities were monitored for a longer period to show intra-participant consistency of the approach (8 weeks of experimentation). An experiment was conducted by involving more participants to demonstrate inter-participant consistency of the approach (7 participants). The reliability of the approach was further demonstrated by comparing results of the experiment with observations of three human judges.

Furthermore, activity verification was put into practice by proposing context-aware applications that support aging in place. Two examples of applications were presented. The first one reminds the user of missed activities with respect to declared routines. The 
second one proposes to send a report assessing the activities of the user to a caregiver or a health professional. Both applications have been run on sensor log data to assess their resulting user interactions and their potential benefits.

Future works will focus on going beyond the simulation of applications based on activity verification. In particular, one goal is to conduct an experiment to assess the accessibility, usability, and acceptability of applications based on activity verification.

Another perspective for activity verification is screening. This line of work leverages the intra-participant consistency of activity verification demonstrated in this paper. The evolution of daily routines and their patterns over several months are key indicators to assess the changes of the functional status of older adults. Activity information could be analyzed by leveraging known patterns of deterioration related to aging diseases. In particular, this information could be used by such health professionals as occupational therapists and geriatricians, specialized in analyzing activities of older adults, and prescribers of assistive support.

\section{Acknowledgements}

We thank the members of the Phoenix research group at Inria for their contributions to this project. Our project was funded by the following partners in France: Caisse Nationale de Solidarité pour l'Autonomie, Chambre des Métiers de la Gironde, Conseil Général de la Gironde, Conseil Régional d’Aquitaine, Union Départementale des Centres Communaux d'Action Sociale de Gironde.

\section{References}

[1] E.D. Mynatt, A.-S. Melenhorst, A.-D. Fisk, and W.A. Rogers, Aware technologies for aging in place: Understanding user needs and attitudes, IEEE Pervasive Computing. 3 (2004) 36-41. doi:10.1109/MPRV.2004.1316816.

[2] B.C. Williams, B.E. Fries, W.J. Foley, D. Schneider, and M. Gavazzi, Activities of daily living and costs in nursing homes, Health Care Financing Review. 15 (1994) 117-135.

[3] E. Pavolini, and C. Ranci, Restructuring the welfare state: reforms in long-term care in Western European countries, Journal of European Social Policy. 18 (2008) 246-259. doi:10.1177/0958928708091058.

[4] D.G. Morrow, and W.A. Rogers, Environmental support: An integrative framework, Human Factors. 50 (2008) 589613. doi:10.1518/001872008X312251.

[5] U. Lindenberger, M. Lövdén, M. Schellenbach, S.-C. Li, and A. Krüger, Psychological principles of successful aging technologies: A mini-review, Gerontology. 54 (2008) 5968. doi:10.1159/000116114.
[6] J. Reijnders, C. van Heugten, and M. van Boxtel, Cognitive interventions in healthy older adults and people with mild cognitive impairment: A systematic review, Ageing $R e$ search Reviews. $12 \quad$ (2013) 263-275. doi:10.1016/j.arr.2012.07.003.

[7] L. Bäckman, and R.A. Dixon, Psychological compensation: A theoretical framework., Psychological Bulletin. 112 (1992) 259-283. doi:10.1037/0033-2909.112.2.259.

[8] A. Gillespie, C. Best, and B. O’Neill, Cognitive function and assistive technology for cognition: A systematic review, Journal of the International Neuropsychological Society. 18 (2012) 1-19. doi:10.1017/S1355617711001548.

[9] J.A. Kaye, S.A. Maxwell, N. Mattek, T.L. Hayes, H. Dodge, M. Pavel, H.B. Jimison, K. Wild, L. Boise, and T.A. Zitzelberger, Intelligent systems for assessing aging changes: Home-based, unobtrusive, and continuous assessment of aging, The Journals of Gerontology Series B: Psychological Sciences and Social Sciences. 66B (2011) i180i190. doi:10.1093/geronb/gbq095.

[10] M. Mokhtari, H. Aloulou, T. Tiberghien, J. Biswas, D. Racoceanu, and P. Yap, New trends to support independence in persons with mild dementia-a mini-review, Gerontology. 58 (2012) 554-563. doi:10.1159/000337827.

[11] M.A. Hossain, Perspectives of human factors in designing elderly monitoring system, Computers in Human Behavior. 33 (2014) 63-68. doi:10.1016/j.chb.2013.12.010.

[12] M. Philipose, K.P. Fishkin, M. Perkowitz, D.J. Patterson, D. Fox, H. Kautz, and D. Hahnel, Inferring activities from interactions with objects, Pervasive Computing, IEEE. 3 (2004) 50-57.

[13] B. Logan, J. Healey, M. Philipose, E.M. Tapia, and S. Intille, A Long-Term Evaluation of Sensing Modalities for Activity Recognition, in: J. Krumm, G.D. Abowd, A. Seneviratne, and T. Strang (Eds.), UbiComp 2007: Ubiquitous Computing, Springer Berlin Heidelberg, Berlin, Heidelberg, 2007: pp. 483-500. doi:10.1007/978-3-540-74853-3_28.

[14] L. Caroux, C. Consel, L. Dupuy, and H. Sauzéon, Verification of daily activities of older adults: A simple, non-intrusive, low-cost approach, in: Proceedings of the 16th International ACM SIGACCESS Conference on Computers \& Accessibility, ACM, New York, NY, USA, 2014: pp. 4350. doi:10.1145/2661334.2661360.

[15] V. Bergua, J. Bouisson, J.-F. Dartigues, J. Swendsen, C. Fabrigoule, K. Pérès, and P. Barberger-Gateau, Restriction in instrumental activities of daily living in older persons: Association with preferences for routines and psychological vulnerability, The International Journal of Aging and Hu$\begin{array}{llll}\text { man Development. } & 77 & \text { (2013) 309-329. }\end{array}$ doi:10.2190/AG.77.4.c.

[16] M.P. Lawton, Aging and performance of home tasks, $\mathrm{Hu}$ $\begin{array}{llll}\text { man } & \text { Factors. } & 32 & \text { (1990) }\end{array}$ doi:10.1177/001872089003200503.

[17] M.C. Clark, S.J. Czaja, and R.A. Weber, Older adults and daily living task profiles, Human Factors. 32 (1990) 537549. doi:10.1177/001872089003200504.

[18] A.D. Fisk, W.A. Rogers, N. Charness, S.J. Czaja, and J. Sharit, Designing for older adults: Principles and creative human factors approaches, 2nd ed, CRC Press, Boca Raton, 2009.

[19] D.A. Gold, An examination of instrumental activities of daily living assessment in older adults and mild cognitive impairment, Journal of Clinical and Experimental Neuro$\begin{array}{llll}\text { psychology. } & 34 & \text { (2012) }\end{array}$ doi:10.1080/13803395.2011.614598

[20] L. Chen, C.D. Nugent, and H. Wang, A knowledge-driven approach to activity recognition in smart homes, IEEE 
Transactions on Knowledge and Data Engineering. 24 (2012) 961-974. doi:10.1109/TKDE.2011.51.

[21] J.C. Allaire, and M. Marsiske, Well-and ill-defined measures of everyday cognition: relationship to older adults' intellectual ability and functional status, Psychology and Aging. 17 (2002) 101-115. doi:10.1037/08827974.17.1.101.

[22] T. Salthouse, Consequences of age-related cognitive declines, Annual Review of Psychology. 63 (2012) 201-226. doi:10.1146/annurev-psych-120710-100328.

[23] J. Bouisson, Routinization preferences, anxiety, and depression in an elderly French sample, Journal of Aging Studies. 16 (2002) 295-302. doi:10.1016/S0890-4065(02)00051-8.

[24] A.M. Seelye, M. Schmitter-Edgecombe, D.J. Cook, and A. Crandall, Naturalistic assessment of everyday activities and prompting technologies in mild cognitive impairment., Journal of the International Neuropsychological Society: JINS. 19 (2013) 442-52. doi:10.1017/S135561771200149X

[25] S.H. Fischer, D. David, B.H. Crotty, M. Dierks, and C. Safran, Acceptance and use of health information technology by community-dwelling elders, International Journal of Medical Informatics. 83 (2014) 624-635. doi:10.1016/j.ijmedinf.2014.06.005.

[26] A.J. Bharucha, V. Anand, J. Forlizzi, M.A. Dew, C.F. Reynolds III, S. Stevens, and H. Wactlar, Intelligent assistive technology applications to dementia care: current capabilities, limitations, and future challenges, The American Journal of Geriatric Psychiatry. 17 (2009) 88-104. doi:10.1097/JGP.0b013e318187dde5.

[27] K.E. Caine, A.D. Fisk, and W.A. Rogers, Benefits and privacy concerns of a home equipped with a visual sensing system: A perspective from older adults, Proceedings of the Human Factors and Ergonomics Society Annual Meeting. 50 (2006) 180-184. doi:10.1177/154193120605000203.

[28] N. Roy, A. Misra, and D. Cook, Ambient and smartphone sensor assisted ADL recognition in multi-inhabitant smart environments, Journal of Ambient Intelligence and Humanized Computing. 7 (2016) 1-19. doi:10.1007/s12652-0150294-7.

[29] L. Chen, J. Hoey, C.D. Nugent, D.J. Cook, and Zhiwen Yu, Sensor-based activity recognition, IEEE Transactions on Systems, Man, and Cybernetics, Part C (Applications and Reviews). $42 \quad$ (2012) 790-808. doi:10.1109/TSMCC.2012.2198883.

[30] S.T.M. Peek, E.J.M. Wouters, J. van Hoof, K.G. Luijkx, H.R. Boeije, and H.J.M. Vrijhoef, Factors influencing acceptance of technology for aging in place: A systematic review, International Journal of Medical Informatics. 83 (2014) 235-248. doi:10.1016/j.ijmedinf.2014.01.004.

[31] A. Mihailidis, J.N. Boger, T. Craig, and J. Hoey, The $\mathrm{COACH}$ prompting system to assist older adults with dementia through handwashing: An efficacy study, BMC Geriatrics. 8 (2008) 28. doi:10.1186/1471-2318-8-28.

[32] M. Marsiske, and J.A. Margrett, Everyday problem solving and decision making, Handbook of the Psychology of Aging. 6 (2006) 315-342.

[33] G. Amato, D. Bacciu, S. Chessa, M. Dragone, C. Gallicchio, C. Gennaro, H. Lozano, A. Micheli, G.M.P. O'Hare, A. Renteria, and C. Vairo, A Benchmark Dataset for Human Activity Recognition and Ambient Assisted Living, in: $\mathrm{H}$. Lindgren, J.F. De Paz, P. Novais, A. Fernández-Caballero, H. Yoe, A. Jiménez Ramírez, and G. Villarrubia (Eds.), Ambient Intelligence- Software and Applications - 7th International Symposium on Ambient Intelligence (ISAmI 2016), Springer International Publishing, Cham, 2016: pp. 1-9. doi:10.1007/978-3-319-40114-0_1.
[34] J. Rafferty, C.D. Nugent, J. Liu, and L. Chen, From activity recognition to intention recognition for assisted living within smart homes, IEEE Transactions on Human-Machine Systems. $47 \quad$ (2017) 368-379. doi:10.1109/THMS.2016.2641388.

[35] K. Yordanova, and T. Kirste, A process for systematic development of symbolic models for activity recognition, ACM Transactions on Interactive Intelligent Systems. 5 (2015) 1-35. doi:10.1145/2806893.

[36] P.N. Dawadi, D.J. Cook, M. Schmitter-Edgecombe, and C. Parsey, Automated assessment of cognitive health using smart home technologies, Technology and Health Care. (2013) 323-343. doi:10.3233/THC-130734.

[37] P.N. Dawadi, D.J. Cook, and M. Schmitter-Edgecombe, Automated cognitive health assessment using smart home monitoring of complex tasks, IEEE Transactions on Systems, Man, and Cybernetics: Systems. 43 (2013) 1302-1313. doi:10.1109/TSMC.2013.2252338

[38] V. Bergua, and J. Bouisson, Vieillesse et routinisation : une revue de la question, Psychologie et Neuropsychiatrie $\mathrm{Du}$ $\begin{array}{llll}\text { Vieillissement. } & 6 & \text { (2008) } & \text { 235-243. }\end{array}$ doi:10.1684/pnv.2008.0144.

[39] M.F. Folstein, S.E. Folstein, and P.R. McHugh, Mini-mental state: a practical method for grading the cognitive state of patients for the clinician, Journal of Psychiatric Research 12 (1975) 189-198. doi:10.1016/0022-3956(75)90026-6.

[40] C. Owsley, M. Sloane, G. McGwin Jr, and K. Ball, Timed instrumental activities of daily living tasks: Relationship to cognitive function and everyday performance assessments in older adults, Gerontology. 48 (2002) 254-265. doi:10.1159/000058360.

[41] M.P. Lawton, M. Moss, M. Fulcomer, and M.H. Kleban, A research and service oriented multilevel assessment instrument, Journal of Gerontology. 37 (1982) 91-99. doi:10.1093/geronj/37.1.91.

[42] W. Donaldson, Measuring recognition memory., Journal of Experimental Psychology: General. 121 (1992) 275-277. doi:10.1037/0096-3445.121.3.275

[43] H. Stanislaw, and N. Todorov, Calculation of signal detection theory measures, Behavior Research Methods, Instruments, \& Computers. 31 (1999) 137-149. doi:10.3758/BF03207704.

[44] S. Lauriks, A. Reinersmann, H.G. Van der Roest, F.J.M. Meiland, R.J. Davies, F. Moelaert, M.D. Mulvenna, C.D. Nugent, and R.M. Dröes, Review of ICT-based services for identified unmet needs in people with dementia, Ageing Re$\begin{array}{lllll}\text { search Reviews. } 6 \text { (2007) 223-246. } & 6\end{array}$ doi:10.1016/j.arr.2007.07.002.

[45] M.R. McGee-Lennon, M.K. Wolters, and S. Brewster, User-centred multimodal reminders for assistive living, in: Proceedings of the SIGCHI Conference on Human Factors in Computing Systems CHI'11, ACM Press, 2011: p. 2105. doi:10.1145/1978942.1979248.

[46] K.M. Wolters, K.-P. Engelbrecht, F. Gödde, S. Möller, A. Naumann, and R. Schleicher, Making it easier for older people to talk to smart homes: The effect of early help prompts, Universal Access in the Information Society. 9 (2010) 311325. doi:10.1007/s10209-009-0184-x.

[47] E. de Joode, C. van Heugten, F. Verhey, and M. van Boxtel, Efficacy and usability of assistive technology for patients with cognitive deficits: A systematic review, Clinical Rehabilitation. $\quad 24 \quad$ (2010) 701-714. doi:10.1177/0269215510367551. 
[48] E. Frank Lopresti, A. Mihailidis, and N. Kirsch, Assistive technology for cognitive rehabilitation: State of the art, $\mathrm{Neu}$ ropsychological Rehabilitation. 14 (2004) 5-39. doi:10.1080/09602010343000101.

[49] M.L. Lee, and A.K. Dey, Embedded assessment of aging adults: A concept validation with stakeholders, in: 4th International Conference on Pervasive Computing Technologies for Healthcare (PervasiveHealth), IEEE, 2010: pp. 1-8. doi:10.4108/ICST.PERVASIVEHEALTH2010.8849.

[50] L.L. Huber, K. Shankar, K. Caine, K. Connelly, L.J. Camp, B.A. Walker, and L. Borrero, How in-home technologies mediate caregiving relationships in later life, International Journal of Human-Computer Interaction. 29 (2013) 441455. doi:10.1080/10447318.2012.715990. 